\title{
Sirolimus-Based Therapy Following Early Cyclosporine Withdrawal Provides Significantly Improved Renal Histology and Function at 3 Years
}

\author{
Alfredo Mota ${ }^{a, *}$, Manuel Arias ${ }^{b}$, Eero I. \\ Taskinenc, Timo Paavonen ${ }^{c, d}$, Yves Brault ${ }^{\mathrm{e}}$, \\ Christophe Legendre ${ }^{f}$, Kerstin Claessong, Marco \\ Castagneto $^{h}$, Josep M. Campistol', Brian \\ Hutchison', James T. Burke ${ }^{\mathrm{e}}$, Sedar Yilmazk, \\ Pekka Häyryc and John F. Neylan', for the \\ Rapamune Maintenance Regimen Trial

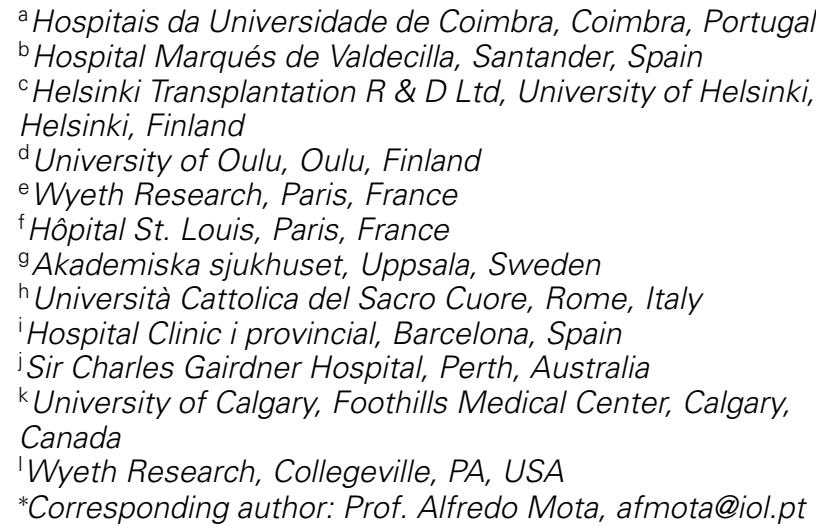

Graft function and histology are predictive of renal transplant survival. The Rapamune Maintenance Regimen study demonstrated that early cyclosporine (CsA) withdrawal from a sirolimus (SRL)-CsA-steroid (ST) regimen improved renal function and blood pressure. We report the protocol-mandated biopsy findings from that study. Renal transplant patients $(n=430)$ receiving SRL-CsA-ST were randomized at 3 months after transplantation to remain on SRL-CsA-ST, or to have CsA withdrawn (SRL-ST group). Protocol-mandated biopsies were performed at engraftment and at 12 and 36 months. Two pathologists blindly evaluated 484 biopsies to obtain the Chronic Allograft Damage Index (CADI) scores. At $\mathbf{3 6}$ months among patients with serial biopsies ( $n=63$ ), the mean CADI score was significantly lower with SRL-ST(4.70 vs. $3.20, p=0.003)$, as was the mean tubular atrophy score $(0.77$ vs. $0.32, p<$ 0.001). All six components of the CADI score were numerically lower in SRL-ST group; moreover, inflammation and the tubular atrophy scores decreased significantly in the SRL-ST group between 12 and 36 months. The calculated glomerular filtration rate at $\mathbf{3 6}$ months was significantly better in the CsA-withdrawal group (54.8 vs. $68.2 \mathrm{~mL} / \mathrm{min}, p=0.009$ ). In conclusion, with- drawing CsA from the SRL-CsA-ST regimen resulted in improved renal histology and function.

Key words: Kidney transplantation, protocol biopsy, renal function, sirolimus

Received 18 November 2003, revised and accepted for publication 10 February 2004

\section{Introduction}

The introduction of new therapeutic options in the treatment of renal transplant recipients offers an opportunity to seek out and validate new and more relevant predictors of long-term patient and graft survival. Recent epidemiologic studies $(1,2)$, predominantly evaluating cyclosporine (CsA)based regimens, have shown that both 12-month serum creatinine and the changes in creatinine during the first year after transplantation are predictive of long-term graft survival. There is also experimental and clinical evidence that renal histologic findings can be quantitatively scored using the Chronic Allograft Damage Index (CADI) to predict progression to chronic rejection and graft loss in renal transplantation $(3,4)$. Clinical trials utilizing quantitative measures of both renal function and histologic data may be especially important in the assessment of immunosuppressive agents.

The calcineurin inhibitors (CNIs), CsA and tacrolimus, have contributed to the low acute rejection rates and favorable 12-month graft survival rates attained today in renal transplantation. Refinements in their use have also been associated with an improvement in graft half-life in recent years, although this improvement has been most marked in a subset of patients capable of maintaining good renal function (serum creatinine $<1.5 \mathrm{mg} / \mathrm{dL}$ ) at 1 year despite $\mathrm{CNI}$ therapy (1). Clearly, these results are suboptimal and even worrisome when confronted with the growing shortage of donors and the declining quality of donor organs for renal transplantation.

Despite their efficacy, CNIs contribute to suboptimal renal graft half-life because they increase blood pressure, decrease glomerular filtration rates (GFR), and contribute to chronic allograft nephropathy (CAN) (5). They also damage 
native kidneys in nonrenal organ transplantation (6). The nephrotoxicity of CNIs may be difficult to differentiate from chronic rejection. Histologic characteristics of $\mathrm{CNI}$-induced nephrotoxicity include tubular vacuolization, microvascular toxicity including arteriolar hyalinization, and stripped or diffuse interstitial fibrosis (7).

Sirolimus (SRL), a mammalian target of rapamycin (mTOR) inhibitor, is not directly nephrotoxic $(8,9)$, although both sirolimus and its derivative, everolimus, enhance the nephrotoxicity of CsA $(10,11)$. The Rapamune Maintenance Regimen (RMR) study has shown that early elimination of CsA from a SRL-CsA-steroid (ST) regimen resulted in significantly better renal function and blood pressure, with a growing advantage in graft survival when compared with a continuous SRL-CSA-ST regimen (12-14). These findings were observed despite numerically more postrandomization rejections in the CsA withdrawal arm (5.6\% vs. 10.2\%, $p=0.107$ ) (14). In the present paper, we report the histologic findings from the protocol-mandated biopsies obtained from renal transplant recipients enrolled in the RMR study.

\section{Materials and Methods}

\section{Study design}

Details concerning the design of the RMR study have been previously described (12). To summarize, 525 patients enrolled at 57 centers received sirolimus $2 \mathrm{mg} /$ day (tablet formulation), CsA, and steroids from the time of transplantation. The demographic features $164.0 \%$ male gender, $95.4 \%$ White race, $89.1 \%$ cadaveric donor sourced with a mean donor age of 43.8 years) were representative and typical for the countries in which the study was carried out (Europe, Canada and Australia). Sirolimus doses were adjusted to maintain whole blood trough levels $>5 \mathrm{ng} / \mathrm{mL}$ (immunoassay). Eligible patients $(n=430)$ were randomly assigned at month 3 ( 2 weeks after transplantation to one of two treatment groups (SRL-CSA-ST or SRLST). Patients continuing on triple therapy (SRL-CsA-ST, $n=215$ ) received the same sirolimus dose (nominally $2 \mathrm{mg} /$ day, troughs $>5 \mathrm{ng} / \mathrm{mL}$ ), CsA (troughs $75-200 \mathrm{ng} / \mathrm{mL}$ from randomization through 24 months, and 50$150 \mathrm{ng} / \mathrm{mL}$ thereafter), and steroids. In the CsA withdrawal group (SRL-ST, $\mathrm{n}=215$ ), sirolimus doses were increased to attain trough concentrations of $20-30 \mathrm{ng} / \mathrm{mL}$ (immunoassay) through month 12 , and $15-25 \mathrm{ng} / \mathrm{mL}$ thereafter.

With the exception of two sites that opted out by protocol amendment, all patients were to undergo protocol biopsies at the time of engraftment (baseline) and at 12 and 36 months after transplantation, providing that they were on assigned therapy at these time points. For each biopsy, at least three stained slides were to be provided (one stain each of hematoxylin-eosin, Masson's trichrome, and periodic acid-Schiff [D-PAS]), plus a minimum of two unstained slides. Wyeth Research collected all biopsy slides for sorting and verification of the labeling before shipping them to the central pathology facility. All slides were labeled with the patient number and the time of biopsy (baseline, 12 months, or 36 months).

\section{Histologic quantitation}

Two pathologists (E.I.P. and T.P.) at the central pathology facility (Helsinki Transplantation R \& D Ltd, University of Helsinki, Finland) independently evaluated each set of biopsy slides. The pathologists remained blinded to treatment and clinical outcome. They rated each of the six components of the CADI score (diffuse or focal inflammation, tubular atrophy, intimal proliferation, glomerular sclerosis, mesangial matrix increase, and interstitial fibrosis) from 0 to 3 using increments of 0.5 (i.e. $0,0.5,1.0,1.5,2.0,2.5$, 3.0). These components correspond to the ' $i$ ', 'ct', ' $\mathrm{cv}^{\prime}$ ' ' $\mathrm{cg}$ ', ' $\mathrm{mm}$ ', and 'ci' scores according to the Banff 97 classification system (7). Details about the scoring criteria for the CADI components are described elsewhere (15).

Specimens were considered adequate if there were seven or more glomeruli, marginal if there were 1-6 glomeruli, and unsatisfactory if there were no glomeruli. Only unsatisfactory biopsies were excluded. The readings from the two pathologists were compared, and CADI scores (sum of the six component scores) within a $\pm 15 \%$ deviation of the midpoint were reported as the mean. If the two CADI scores deviated more than $15 \%$ from the midpoint, the two pathologists assessed the slides together to obtain a consensus reading.

\section{Statistical analyses}

The Biostatistics Department of Wyeth Research (Paris, France) performed all statistical analyses. For demography, categorical variables such as sex and ethnic origin were analyzed using the Pearson Chi-square test. Continuous variables such as recipient age and baseline creatinine were compared using analysis of variance (ANOVA) with treatment as a factor. The CADI score, component scores, serum creatinine, and calculated glomerular filtration rate (GFR) (16) were analyzed by analysis of covariance (ANCOVA) with treatment as a fixed factor and baseline as a covariate. Baselines for serum creatinine and calculated GFR were the last values before randomization. For tubular atrophy, the degree of change between 12 and 36 months was also analyzed by a Mantel-Haenszel Chi-square test. Treatment effect was only analyzed for paired biopsy readings (e.g. baseline and 12 months).

\section{Results}

\section{All renal biopsies}

Of the 484 slide sets sent to the pathology facility, 29(6.0\%) were unsatisfactory, $71(14.7 \%)$ were marginal, and 384 $(79.3 \%)$ were satisfactory. Of the $455(94.0 \%)$ sets that were either marginal or satisfactory, $400(87.9 \%)$ of the readings were based on the average of the two pathologists' evaluations and 55 (12.1\%) were consensus readings. A further 174 (38.2\%) baseline biopsies were not forwarded to the central pathology facility because neither a subsequent 12-month nor a 36-month biopsy was received for these patients. The mean $( \pm S D)$ times from first study drug intake to biopsy were $0.07 \pm 1.62$ vs. $0.05 \pm 0.90$ days at baseline, $366.87 \pm 22.50$ vs. $372.76 \pm 22.70$ days at 12 months, and $1102.00 \pm 63.16$ vs. $1108.63 \pm 18.73$ days at 36 months, for SRL-CsA-ST vs. SRL-ST, respectively.

Table 1 summarizes the number of patients and the mean CADI scores at baseline, 12 months, and 36 months. Overall, $54.0 \%$ and $38.6 \%$ of all randomized patients remaining on therapy underwent protocol-mandated biopsies at 12 and 36 months, respectively. Protocol-mandated biopsies were required only in patients on therapy and active within the study. The principal reason for not obtaining a biopsy was patient refusal. Complete serial biopsies were obtained at baseline, 12 , and 36 months in $25.6 \%$ of the on-therapy patients, or $14.6 \%$ of all randomized patients. 
Improved Renal Biopsy Findings with Sirolimus

Table 1: Summary of baseline, 12-month, and 36-month Chronic Allograft Damage Index (CADI) scores

\begin{tabular}{|c|c|c|c|c|c|c|}
\hline \multirow[b]{2}{*}{ Cohort $^{1}$} & \multicolumn{2}{|l|}{ Baseline } & \multicolumn{2}{|l|}{12 months } & \multicolumn{2}{|l|}{36 months } \\
\hline & SRL-CsA-ST & SRL-ST & SRL-CsA-ST & SRL-ST & SRL-CsA-ST & SRL-ST \\
\hline \multicolumn{7}{|c|}{ All patients with biopsy readings } \\
\hline Mean \pm SD & $1.27 \pm 1.37$ & $1.23 \pm 1.36$ & $3.56 \pm 1.92$ & $3.45 \pm 1.79$ & $4.39 \pm 1.84$ & $3.59 \pm 1.98$ \\
\hline $\begin{array}{l}\text { Range } \\
\mathrm{n}\end{array}$ & $\begin{array}{l}0.00-8.33 \\
(97)^{1}\end{array}$ & $\begin{array}{l}0.00-8.00 \\
(82)\end{array}$ & $\begin{array}{l}0.25-8.50 \\
(95)\end{array}$ & $\begin{array}{l}0.00-8.25 \\
(86)\end{array}$ & $\begin{array}{l}0.75-8.00 \\
(47)\end{array}$ & $\begin{array}{l}0.50-9.00 \\
(48)\end{array}$ \\
\hline \multicolumn{7}{|c|}{ Biopsies at baseline and 12 months } \\
\hline Mean \pm SD & $1.27 \pm 1.38$ & $1.15 \pm 1.35$ & $3.64 \pm 1.82$ & $3.63 \pm 1.82$ & & \\
\hline $\begin{array}{l}\text { Range } \\
\mathrm{n}\end{array}$ & $\begin{array}{l}0.00-8.33 \\
(79)\end{array}$ & $\begin{array}{l}0.00-8.00 \\
(68)\end{array}$ & $\begin{array}{l}0.50-8.50 \\
(79)\end{array}$ & $\begin{array}{l}0.00-8.25 \\
(68)\end{array}$ & & \\
\hline \multicolumn{7}{|c|}{ Biopsies at baseline and 36 months } \\
\hline Mean \pm SD & $1.17 \pm 1.51$ & $1.02 \pm 0.89$ & & & $4.50 \pm 1.84^{2}$ & $3.23 \pm 1.84^{2}$ \\
\hline $\begin{array}{l}\text { Range } \\
\mathrm{n}\end{array}$ & $\begin{array}{l}0.00-8.33 \\
(40)\end{array}$ & $\begin{array}{l}0.00-3.33 \\
(37)\end{array}$ & & & $\begin{array}{l}1.25-8.00 \\
(40)\end{array}$ & $\begin{array}{l}0.50-9.00 \\
(37)\end{array}$ \\
\hline \multicolumn{7}{|c|}{ Biopsies at 12 months and 36 months } \\
\hline Mean \pm SD & & & $3.62 \pm 2.04$ & $3.50 \pm 1.65$ & $4.55 \pm 1.92$ & $3.63 \pm 2.02$ \\
\hline $\begin{array}{l}\text { Range } \\
\mathrm{n}\end{array}$ & & & $\begin{array}{l}0.25-8.50 \\
(35)\end{array}$ & $\begin{array}{l}0.75-7.75 \\
(42)\end{array}$ & $\begin{array}{l}0.75-8.00 \\
(35)\end{array}$ & $\begin{array}{l}0.50-9.00 \\
(42)\end{array}$ \\
\hline \multicolumn{7}{|c|}{ Biopsies at baseline, 12 months, and 36 months } \\
\hline Mean \pm SD & $1.26 \pm 1.60$ & $0.99 \pm 0.92$ & $3.74 \pm 1.84$ & $3.53 \pm 1.66$ & $4.70 \pm 1.86^{3}$ & $3.20 \pm 1.85^{3}$ \\
\hline $\begin{array}{l}\text { Range } \\
n\end{array}$ & $\begin{array}{l}0.00-8.33 \\
(31)\end{array}$ & $\begin{array}{l}0.00-3.33 \\
(32)\end{array}$ & $\begin{array}{l}0.75-8.50 \\
(31)\end{array}$ & $\begin{array}{l}0.75-7.75 \\
(32)\end{array}$ & $\begin{array}{l}1.25-8.00 \\
(31)\end{array}$ & $\begin{array}{l}0.50-9.00 \\
(32)\end{array}$ \\
\hline
\end{tabular}

${ }_{1}^{1}$ Patients could be in more than one cohort if they fulfilled the criteria.

${ }^{2} \mathrm{p}=0.004$ for SRL-CsA-ST vs. SRL-ST, ANCOVA change from baseline.

${ }^{3} p=0.003$ for SRL-CsA-ST vs. SRL-ST, ANCOVA change from baseline.

At 12 months, there was no treatment effect in the CADI score for the cohort with paired baseline and 12-month biopsies. By 36 months, however, the CADI score was significantly lower in the SRL-ST patients, when analyzed using all baseline and 36-month paired biopsy readings or when limiting the analysis to patients with complete serial biopsies at baseline and at 12 and 36 months. Because the analysis of the various cohorts produced no findings other than those observed in the cohort of patients undergoing all protocol-mandated biopsies, results from these patients will be provided in the following sections.

\section{Cohort with serial biopsies at baseline and at 12 and 36 months}

Characteristics of patients with and without biopsies: Patients who remained on therapy through 36 months and had serial protocol biopsies were compared with those who did not have biopsies for the principal demographic parameters, baseline renal function, and acute rejection status (Table 2). No significant differences were observed in the SRL-CSA-ST group between patients with biopsies and those without. In the SRL-ST group, patients with biopsies had experienced significantly more acute rejections (21.9\% vs. 8.7\%, p =0.042) and numerically more patients had four HLA mismatches ( $40.6 \%$ vs. $26.9 \%, p=0.148$ ) compared with patients who did not have biopsies performed. Intergroup comparisons of patients with biopsies revealed that the donors in the SRL-CsA-ST group were significantly older (45.1 vs. 37.2 years, $p=0.045$ ), but this age difference was consistent with a similar imbalance present between groups ( $44.0 \mathrm{vs} .41 .7$ years, $p=0.116$ ) at the time of randomization. The same observation can be made with regards to the percentage of donors aged 60 years: $22.6 \%$ vs. $6.3 \%, p=0.083$, in patients with serial biopsies and $16.3 \%$ vs. $9.8 \%, p=0.062$, in the intent-to-treat (ITT) population, SRL-CsA-ST vs. SRL-ST, respectively. Complete demographic data for the ITT population can be found in the original publication (12).

Similar analyses were also performed to compare patients undergoing biopsy with those who did not using the ITT population (215 patients randomized per group), rather than the population completing 36 months of therapy. No intragroup differences were observed for any of the demographic characteristics or for acute rejection status, indicating that the patients with serial biopsies reflected the ITT population for these parameters (data not shown). Conversely, SRL-CsA-ST patients with serial biopsies had better baseline renal function than the SRL-CsAST patients who did not have biopsies performed. The baseline serum creatinine and calculated GFR were 157.0 vs. $137.2 \mu \mathrm{mol} / \mathrm{L}, \mathrm{p}=0.036$, and 55.9 vs. $60.1 \mathrm{~mL} / \mathrm{min}$, $\mathrm{p}=0.202$, in SRL-CsA-ST patients without and with biopsies, respectively. These baseline parameters, however, were similar for the SRL-ST group: 153.8 vs. $150.0 \mu \mathrm{mol} / \mathrm{L}$, $\mathrm{p}=0.717$, and 55.2 vs. $57.3 \mathrm{~mL} / \mathrm{min}, \mathrm{p}=0.504$, in patients without and with biopsies, respectively. Significantly more patients discontinued from the SRL-CsA-ST group before the 36-month visit, and their renal function at the time of discontinuation was worse than in discontinuing SRL-ST patients (calculated GFR 41.6 vs. $52.3 \mathrm{~mL} / \mathrm{min}$, $\mathrm{p}<0.001)$. 
Table 2: Demographic characteristics, acute rejection status, and baseline renal function in patients with serial biopsies at baseline, 12 months, and 36 months compared with patients on therapy at 36 months who did not have a biopsy

\begin{tabular}{|c|c|c|c|c|}
\hline \multirow[b]{2}{*}{ Biopsy } & \multicolumn{2}{|c|}{ SRL-CsA-ST } & \multicolumn{2}{|l|}{ SRL-ST } \\
\hline & $\begin{array}{l}\text { No } \\
(n=93)\end{array}$ & $\begin{array}{l}\text { Yes } \\
(n=31)\end{array}$ & $\begin{array}{l}\text { No } \\
(n=104)\end{array}$ & $\begin{array}{l}\text { Yes } \\
(n=32)\end{array}$ \\
\hline Sex (\% male) & 66.7 & 67.7 & 55.8 & 68.8 \\
\hline Ethnic origin, White (\%) & 93.5 & 96.8 & 96.2 & 100.0 \\
\hline Recipient age (years) & 44.8 & 44.4 & 43.7 & 43.3 \\
\hline Donor age (years) & 40.9 & $45.1^{1}$ & 39.0 & $37.2^{1}$ \\
\hline Cadaver donor $(\%)$ & 88.2 & 83.9 & 92.3 & 93.8 \\
\hline Secondary transplants (\%) & 9.7 & 9.7 & 10.6 & 9.4 \\
\hline Ischemia time, mean (h) & 17.6 & 16.2 & 17.0 & 17.2 \\
\hline HLA mismatches > $3(\%)$ & 28.0 & 29.0 & 26.9 & 40.6 \\
\hline Delayed graft function $(\%)^{3}$ & 17.2 & 12.9 & 19.2 & 21.9 \\
\hline \multicolumn{5}{|l|}{ Incidence acute rejection (\%) } \\
\hline Prerandomization ${ }^{4}$ & 5.4 & 3.2 & 7.7 & 15.6 \\
\hline Posttransplantation, 0-36 months & 7.5 & 6.5 & $8.7^{2}$ & $21.9^{2}$ \\
\hline Serum creatinine at randomization, mean $(\mu \mathrm{mol} / \mathrm{L})$ & 139.7 & 137.2 & 145.2 & 150.0 \\
\hline Calculated GFR at randomization, mean (mL/min) & 61.3 & 60.1 & 56.5 & 57.3 \\
\hline
\end{tabular}

${ }_{1}^{1} p<0.05$ for SRL-CsA-ST vs. SRL-ST in patients with biopsies, ${ }^{2} p<0.05$ for intragroup comparisons of patients with or without biopsies.

${ }^{3}$ Need for dialysis in the first 7 days after transplantation.

${ }^{4}$ Randomization occurred at month $3 \pm 2$ weeks.

In patients with serial biopsies, median sirolimus whole blood trough levels, as measured by monoclonal immunoassay (median daily doses), in the SRL-CsA-ST group were $10.1 \mathrm{ng} / \mathrm{mL}(2.0 \mathrm{mg})$ and $13.1 \mathrm{ng} / \mathrm{mL}(2.0 \mathrm{mg})$ at months 12 and 36 , respectively. In the SRL-ST group, these parameters were $22.3 \mathrm{ng} / \mathrm{mL}(8.0 \mathrm{mg})$ and $17.4 \mathrm{ng} / \mathrm{mL}$ $(5.0 \mathrm{mg})$ at months 12 and 36, respectively. Median CsA whole blood trough levels as measured by monoclonal immunoassay (median daily doses) in the SRL-CsA-ST group were $139.1 \mathrm{ng} / \mathrm{mL}(200 \mathrm{mg})$ and $85.0 \mathrm{ng} / \mathrm{mL}(175 \mathrm{mg})$ at months 12 and 36 , respectively. These values were similar to those in patients without biopsies.

\section{Histologic scores}

Mean CADI scores are presented in Figure 1A. They were comparable between the two treatment groups both at baseline and 12 months, progressing approximately 2.5 units over the first year. The CADI scores diverged thereafter, becoming significantly lower in the SRL-ST group at 36 months ( $p=0.003$ ). The difference between groups was also significant ( $p=0.006$ ) over the 12- to 36-month period. As would be expected following early and complete CsA withdrawal, changes in renal function (Figure 1B) preceded changes in the CADI scores. Nonetheless, even though CSA withdrawal was not completed in all SRL-ST patients until month 5, differences in CADI component scores were already appearing by month 12 .

Among the CADI component scores (Figure 2), the increase in the inflammation score was significantly greater in the SRL-ST group at month 12 (Figure 2A). The increase in inflammation was not significantly different among patients experiencing a rejection in the first 12 months $(0.88$ vs. 0.93, $p=0.778$, SRL-CsA-ST vs. SRL-ST), but it was significantly higher in the SRL-ST group among those who did not have an acute rejection (0.45 vs. $0.78, \mathrm{p}=$ 0.041). Inflammation at 12 months was not accompanied by structural changes as evidenced by Banff 97 tubulitis (t), glomerulitis (g), or arteritis (v) scores (data not shown). Moreover, without any intervention, the mean inflammation score decreased by 0.33 units from month 12 to month 36 in the SRL-ST group. The changes between 12 and 36 months were statistically significant in favor of the SRL-ST group (0.05 vs. $-0.33, p=0.004)$, although the 36-month scores based on the change from baseline analysis were not (0.64 vs. 0.50, SRL-CsA-ST vs. SRL-ST, $p=0.318$ ).

At 36 months, the tubular atrophy score was significantly lower in the SRL-ST group (Figure 2B). Importantly, the mean tubular atrophy score decreased significantly between 12 and 36 months in the SRL-ST group (0.07 vs. $-0.24, p=0.041)$. The distribution of patients with decreasing, stable, or increasing tubular atrophy scores was also significant in favor of CsA withdrawal (Table 3).

The CADI score includes two glomerular components, glomerular sclerosis (Figure 2C) and mesangial matrix increase (Figure 2D). Both of these scores were lower in the SRL-ST group, although neither difference attained statistical significance. Of note, the mean glomerular sclerosis score was unchanged in both groups between baseline and 12 months.

Intimal proliferation was less marked in the SRL-ST group at 12 months and the difference between groups widened during the next 2 years (Figure 2E); however, these differences were not statistically significant. The mean fibrosis score increased approximately 0.8 units in both groups between baseline and 12 months, but remained essentially 
A
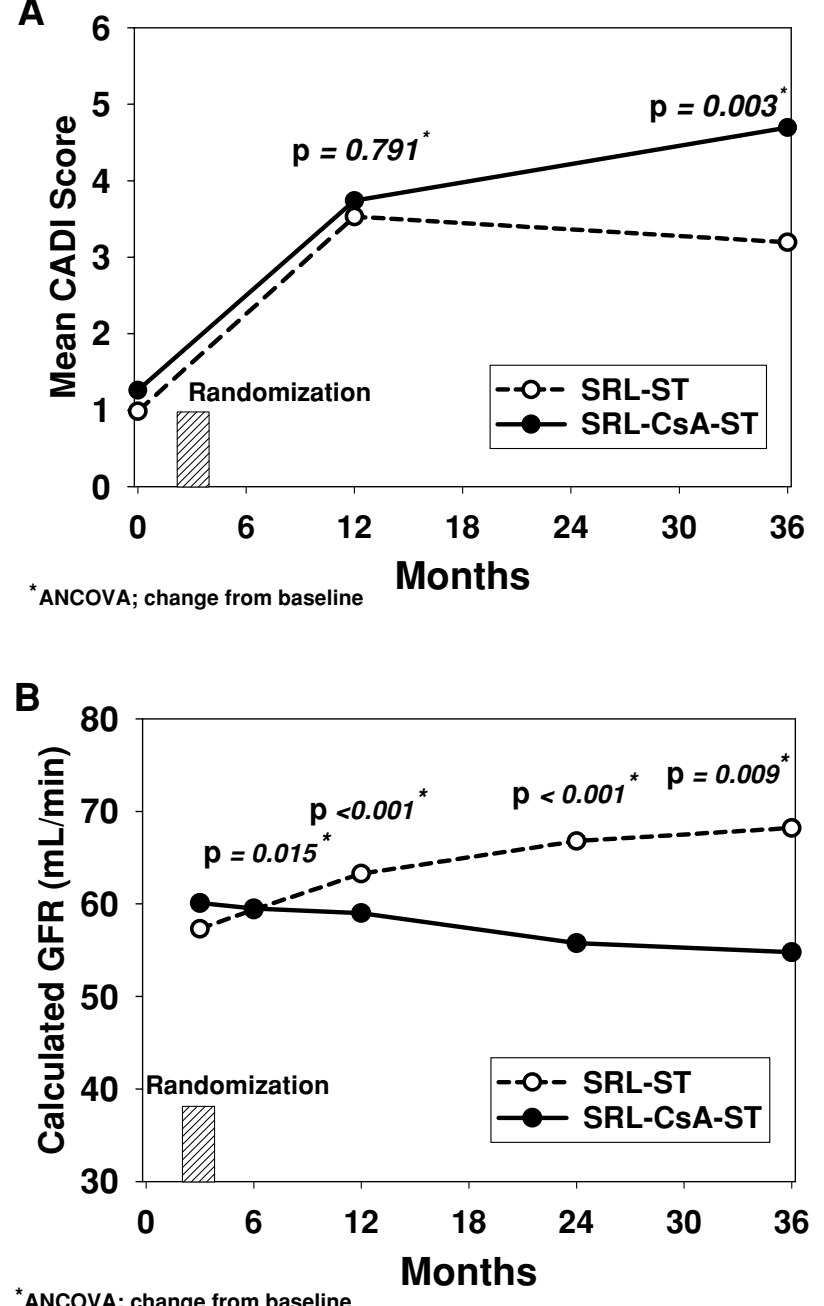

*ANCOVA; change from baseline

Figure 1: Mean Chronic Allograft Damage Index (CADI) score (A) and calculated glomerular filtration rate (GFR) (B) in patients with serial biopsies at baseline, 12 months, and 36 months.

unchanged in both groups over the 12- to 36-month interval (Figure 2F).

\section{Renal function}

Calculated GFR evolved significantly over time to the advantage of CsA withdrawal, irrespective of the population (Table 4). Renal function decreased from the time of randomization in the CsA continuation group, whereas it continued to improve in the SRL-ST group, even after month 12 (Figure 1B). At 36 months, the calculated GFR values as well as the difference between treatments were similar in the cohort of patients undergoing serial biopsies (54.8 vs. $68.2 \mathrm{~mL} / \mathrm{min}, \mathrm{p}=0.009$, SRL-CsA-ST vs. SRL-ST, respectively) when compared with the entire on-therapy population (55.1 vs. $67.0 \mathrm{~mL} / \mathrm{min}, \mathrm{SRL}-\mathrm{CsA}-\mathrm{ST}$ vs. SRL-ST, respectively, $\mathrm{p}<0.001$ ).
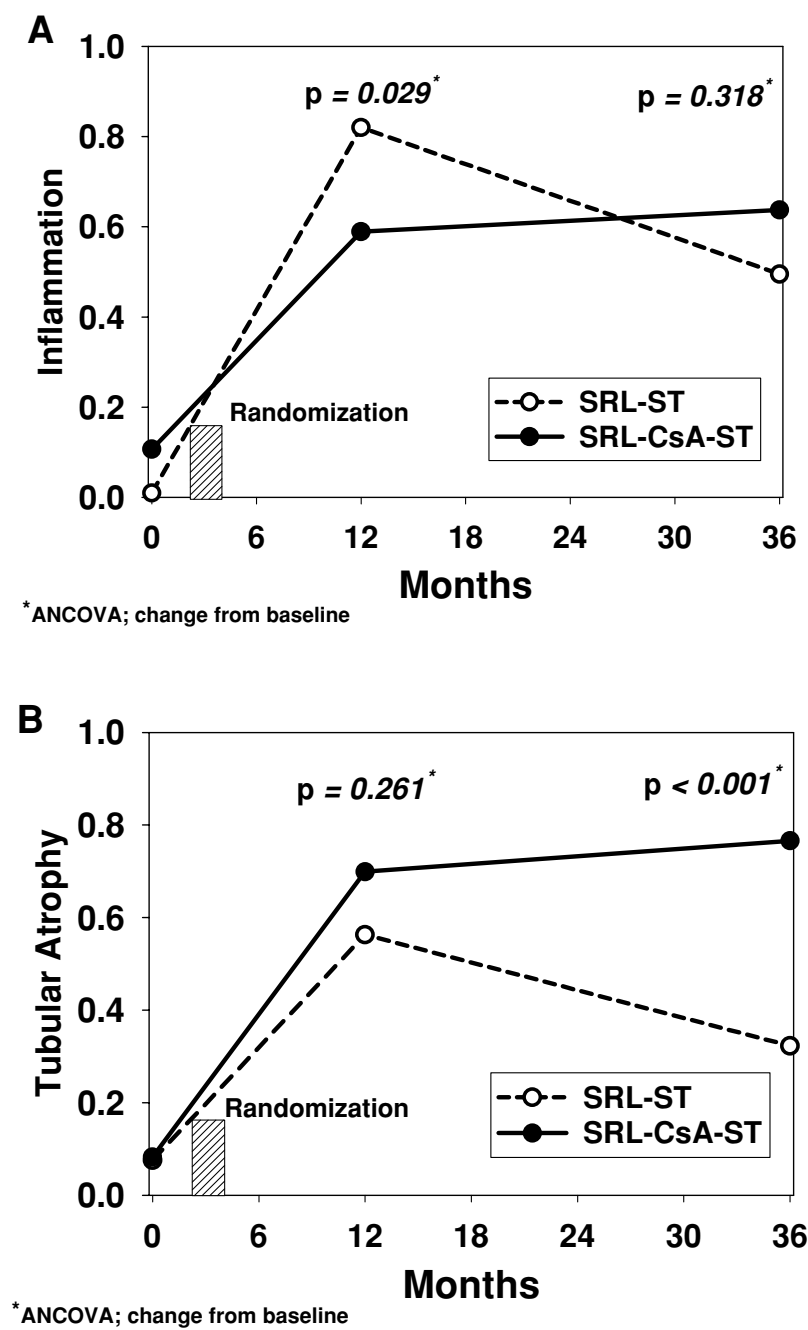

Figure 2: Mean Chronic Allograft Damage Index (CADI) component scores in patients with serial biopsies at baseline, 12 months, and 36 months.

Similar renal function benefit $(12.1 \mathrm{~mL} /$ min difference) was demonstrated in the ITT population at 36 months (47.3 vs. $59.4 \mathrm{~mL} / \mathrm{min}, \mathrm{p}<0.001$, SRL-CsA-ST vs. SRL-ST), but overall, calculated GFR values were lower in the ITT population in both treatment groups when compared with either the cohort of patients with serial biopsies or with the entire on-therapy population. According to biopsy status in the ITT population, calculated GFR at 36 months was 45.9 vs. $54.8 \mathrm{~mL} / \mathrm{min}, \mathrm{p}=0.078$, and 57.7 vs. $68.2 \mathrm{~mL} / \mathrm{min}, \mathrm{p}=$ 0.034 , no biopsy vs. biopsy, in the SRL-CsA-ST and SRL-ST groups, respectively. This indicates that within a group, the mean calculated GFR was approximately $10 \mathrm{~mL} / \mathrm{min}$ lower in patients without serial biopsies compared with those who had biopsies. Intergroup comparisons show that the SRL-ST group fared better, even though this group had a numerically higher incidence of rejection. 


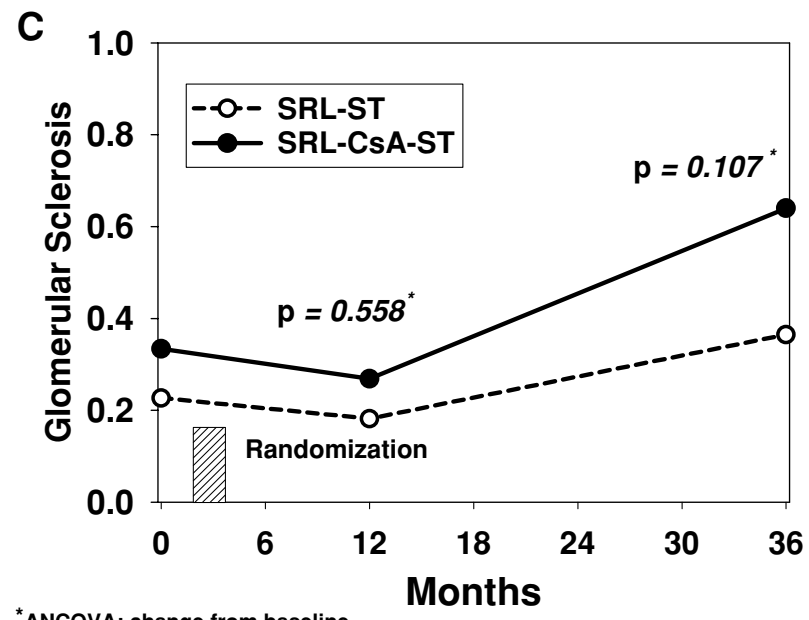

*ANCOVA; change from baseline

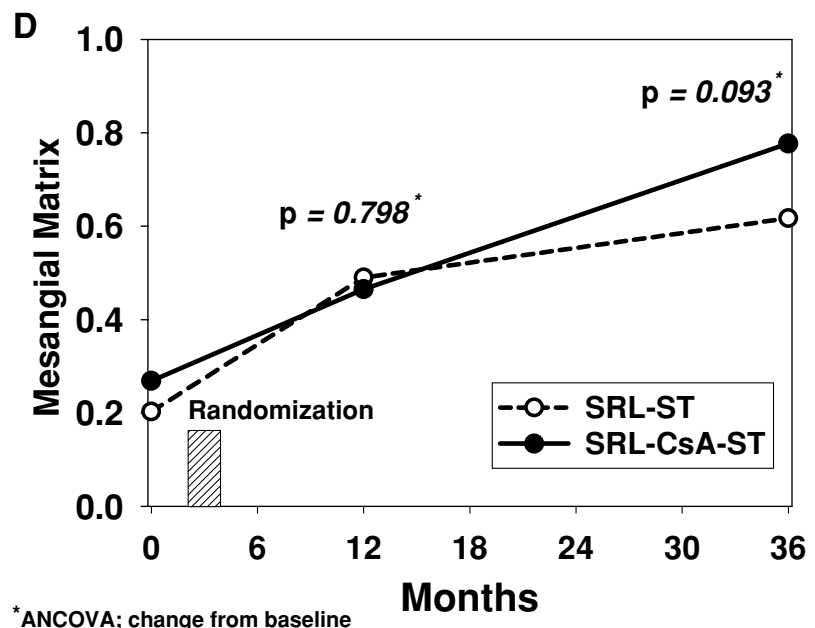

Figure 2: Continued.

Table 3: Change in tubular atrophy scores from 12 to 36 months by change category in patients with serial biopsies at baseline, 12 months, and 36 months

\begin{tabular}{llcl}
\hline & & \multicolumn{2}{c}{ Percentage of patients } \\
\cline { 3 - 4 } \cline { 3 - 4 } Group & $\begin{array}{l}\text { Score } \\
\text { decreased }\end{array}$ & $\begin{array}{l}\text { Score } \\
\text { unchanged }\end{array}$ & $\begin{array}{l}\text { Score } \\
\text { increased }\end{array}$ \\
\hline SRL-CsA+ST & & 9.7 & 54.8 \\
SRL $^{1}$ & 35.5 & 9.7 & 18.8 \\
\hline
\end{tabular}

${ }^{1}$ SRL-CsA-ST vs. SRL-ST, Mantel-Haenszel chi-square (modified ridits) $p$-value $=0.018$

\section{Discussion}

When this study was designed in 1997, we decided to use the numerical CADI scoring for chronic histologic alterations instead of the Banff criteria developed in 1991 (17). At that time, the Banff criteria and threshold values

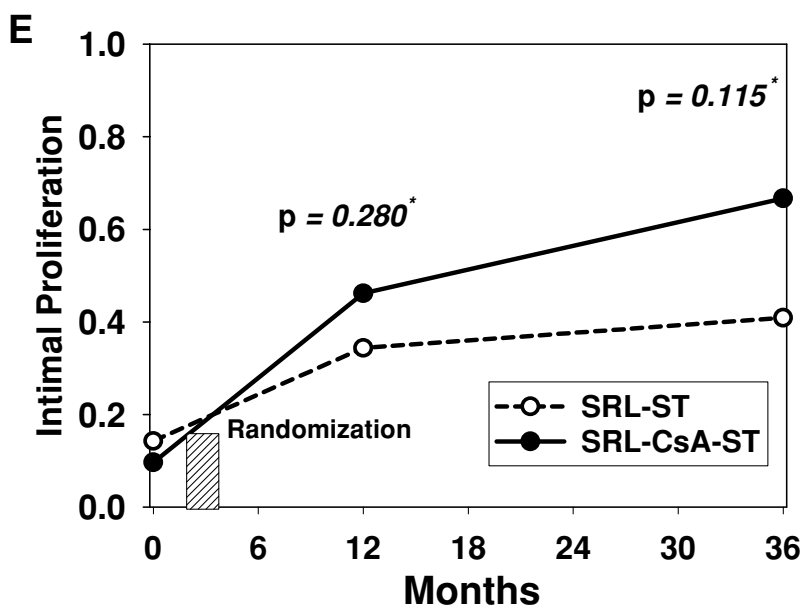

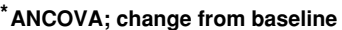

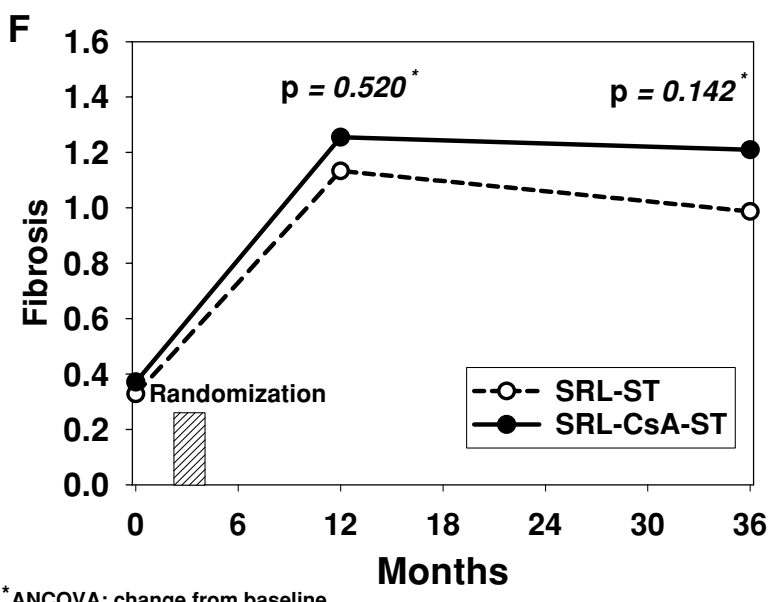

Table 4: Mean ( \pm SEM) calculated GFR ( $\mathrm{mL} / \mathrm{min}$ ) by treatment group at baseline and at 36 months in the ITT population, the on therapy population, and in the population of patients with serial biopsies at baseline, 12 months and 36 months

\begin{tabular}{lllr}
\hline Population & SRL-CsA-ST & SRL-ST & p-value ${ }^{1}$ \\
\hline ITT & & & \\
$\mathrm{n}^{2}$ & 194 & 194 & \\
$\quad$ Baseline & $57.3 \pm 1.2$ & $55.6 \pm 1.2$ & 0.304 \\
$\quad 36$ months & $47.3 \pm 1.8$ & $59.4 \pm 1.8$ & $<0.001$ \\
On therapy & & & \\
$\quad \mathrm{n}$ & 119 & 132 & \\
$\quad$ Baseline & $61.0 \pm 1.4$ & $57.0 \pm 1.4$ & 0.062 \\
$\quad$ 36 months & $55.1 \pm 1.5$ & $67.0 \pm 1.8$ & $<0.001$ \\
Serial biopsies & & & \\
$\quad$ n & 31 & 32 & \\
$\quad$ Baseline & $60.1 \pm 2.0$ & $57.3 \pm 2.7$ & 0.410 \\
$\quad$ 36 months & $54.8 \pm 2.7$ & $68.2 \pm 2.9$ & 0.009 \\
\hline
\end{tabular}

${ }^{1}$ Baseline values compared by ANOVA. Values at 36 months compared by ANCOVA with baseline as covariate.

${ }^{2}$ Exclusive of patients with values missing at month 36 . 
for chronic alterations in biopsy results were not available. Additionally, a single-center study relating the CADI scores in protocol-mandated biopsies with long-term allograft survival had been published (3). Further, an evaluation of biopsies from two mycophenolate mofetil (MMF) studies using exactly the same criteria and being read by the same pathologists was already underway (4). The threshold values for different histologic changes in terms of the six CADI components, however, are similar to the threshold values of the Banff 97 classification, published in 1999 (7). Photomicrographs illustrating the progression of the CADI

\section{A}

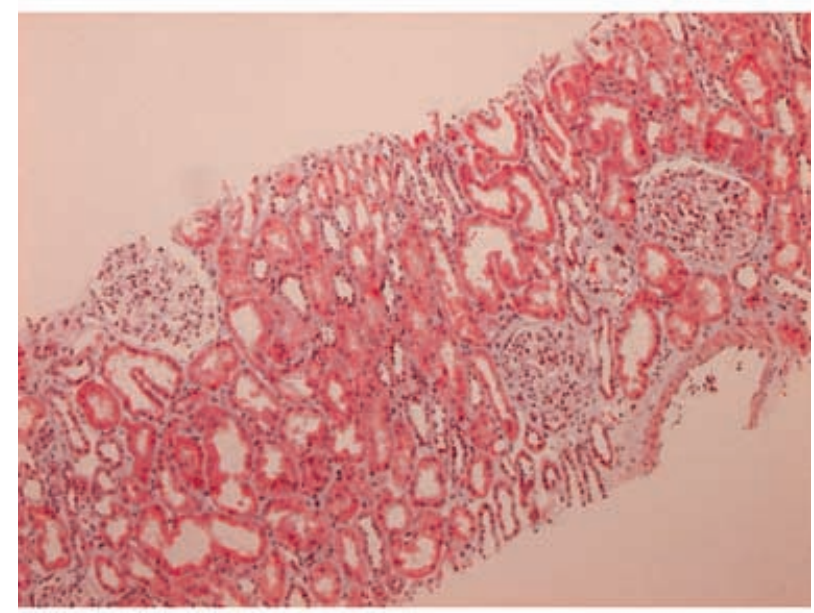

B

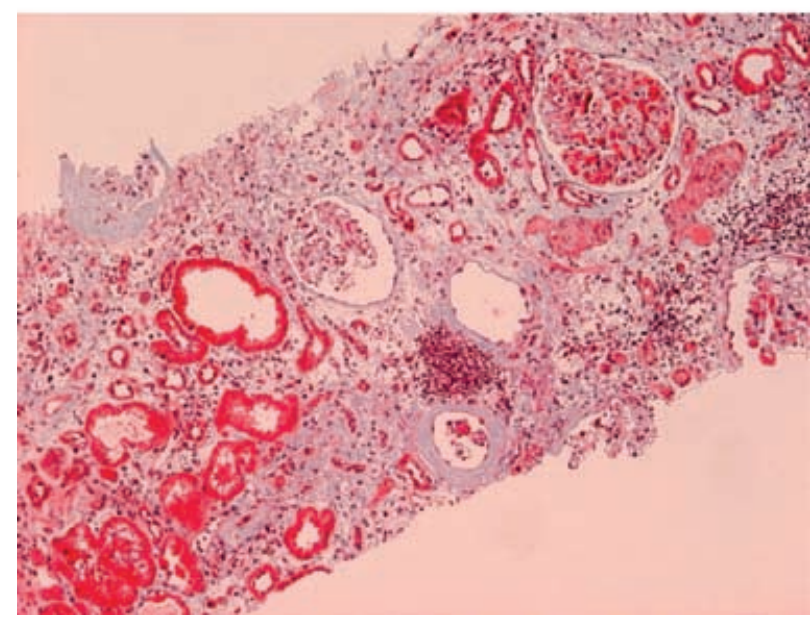

Figure 3: Examples of engraftment (A) and 36-month (B) biopsies scored using Chronic Allograft Damage Index (CADI). (A) Baseline (engraftment) biopsy. Inflammation 0, interstitial fibrosis 0 , tubular atrophy 0 , mesangial matrix 0.5 , glomerular sclerosis 0 , intimal proliferation $0, \mathrm{CADI}=0.5$ (hematoxylin-eosin stain $\times 200$ ). (B) Month 36 biopsy. Inflammation 0.5, interstitial fibrosis 1.5 , tubular atrophy 1.5 , mesangial matrix 1.0 , glomerular sclerosis 0 , intimal proliferation 1.0, CADI $=5.5$. (Masson's trichrome stain $\times 200$ ). score from 0.5 at engraftment to 5.5 at 36 months are presented in Figure 3.

Significantly lower CADI scores in the SRL-ST group at 36 months are consistent with the other principal clinical findings of the RMR trial: improved renal function and lower blood pressure associated with a growing difference in graft survival in favor of early CsA withdrawal. Although biopsies were not obtained in all patients on therapy at 12 and 36 months, the patients undergoing serial biopsies were representative of the entire population of patients on therapy at month 36 . The only significant demographic difference among patients with biopsies was that donors were on average 7.9 years younger in the SRL-ST than in the SRL-CsA-ST group, which was greater than the 2.3-year difference between groups in the entire randomized population. The other statistically significant finding at 36 months was that, among the SRL-ST on-therapy patients, the acute rejection rate was significantly higher in patients undergoing a biopsy compared with those who did not have biopsies performed. Importantly, both baseline and 36-month renal function parameters were similar between on-therapy patients with biopsies and those without biopsies.

Were the patients with serial biopsies representative of the ITT population of randomized patients? No significant differences were observed regarding any of the demographic parameters. In the SRL-CSA-ST group, baseline renal function was better in patients with serial biopsies than in those without serial biopsies. For both treatment groups, however, calculated GFR at 36 months was approximately $10 \mathrm{~mL} / \mathrm{min}$ better in patients undergoing a biopsy than it was among those in the ITT population who did not have a biopsy. This is because patients with declining renal function were more likely to discontinue before 36 months. It should be emphasized, however, that patients in the SRL-CSA-ST group had significantly worse renal function at the time of discontinuation than patients randomized to SRL-ST therapy. With regards to the question at the beginning of this paragraph, patients with serial protocolmandated biopsies had better renal function than those who discontinued before month 36 . This notwithstanding, as discontinuing SRL-CsA-ST patients ( $n=101,47.0 \%)$ had significantly worse renal function than discontinuing SRLST patients ( $n=79,36.7 \%$ ), any bias in favor of the SRL-ST group was highly unlikely.

The CADI score progressed rapidly in both groups between engraftment and 12 months. This finding is consistent with other reports indicating that the histologic sequelae, secondary to a mixture of allogeneic and nonallogeneic factors, occur in up to $25 \%$ of patients, even by month 3 after transplantation (18). It should be emphasized that the baseline biopsies were obtained at engraftment, whereas the patients were randomly assigned at 3 months. The median time to discontinue CsA was 42 days in the SRL-ST group, and the last patient discontinued CsA at 116 days after 
randomization. As a consequence, even patients randomized to SRL-ST were exposed to 4-5 months of CsA use between engraftment and 12-month biopsies.

Despite the fact that the therapies were only different for approximately 7 months during the initial 12 months, differences did emerge at month 12. The inflammation score was significantly higher in the SRL-ST group, whereas the progressions from baseline for the five other components were slightly less in this group compared with SRL-CsA-ST. The net result was that changes from baseline in the CADI score at 12 months were essentially identical between SRL-CsA-ST and SRL-ST (2.48 vs. 2.54, respectively). The significantly higher inflammation score in the SRL-ST group at 12 months was not associated with evidence of tubulitis or glomerulitis. Infiltrates decreased by 36 months in the SRL-ST group without specific antirejection intervention and despite the fact that the sirolimus target and the observed trough levels were $5 \mathrm{ng} / \mathrm{mL}$ lower after month 12 .

The 12- to 36-month interval permits an unambiguous comparison between the two groups. During this interval, all six components of the CADI score evolved in favor of CsA withdrawal. There was a significant decrease in the inflammation score in the SRL-ST group compared with the SRLCsA-ST group ( -0.33 vs. $0.05, p=0.004$ ) so that the mean score at 36 months became numerically lower in that group (0.64 vs. $0.50, p=0.318$ ). At 36 months, the tubular atrophy score was significantly lower in the SRL-ST group. The mean tubular atrophy score also decreased significantly between 12 and 36 months in the SRL-ST group. Among the SRL-ST patients, 53.6\% showed a decrease in their tubular atrophy score between 12 and 36 months, while in $25.0 \%$, the score remained unchanged. Tubular changes are hallmarks of CsA nephrotoxicity. Thus, these data suggest that sirolimus-based, CsA-free therapy will not only reduce the incidence of tubular atrophy, but may also facilitate reversal if $\mathrm{CsA}$ is withdrawn early in the post-transplantation period.

Few large studies have explored treatment-related differences in protocol-mandated biopsy findings. The FK506 Kidney Transplant Study group (19) reported a similar incidence of CAN at 2 years $(72.3 \%$ and $62.0 \%, p=0.155)$ between tacrolimus- and CsA-treated patients, respectively. In that study, the occurrence of CAN was associated with older donor age, presumed CSA- or tacrolimus-induced nephrotoxicty, development of CMV infection, and acute rejection during the first year. Of note in the present study, better renal function and histologic findings were observed in the SRL-ST group despite a higher incidence of acute rejection.

Yilmaz and colleagues reported the biopsy findings from two pivotal MMF studies (4). The results were not analyzed for treatment effects; however, they did conclude that the CADI score at 1 year was predictive of subsequent graft loss and was therefore a good surrogate marker for long-term graft survival in multicenter studies. Their report is of particular interest in the context of the present study because the same two pathologists who read the biopsies for the MMF studies also evaluated them for the RMR trial, using the exact same methodology. In the MMF studies, 43 patients underwent serial biopsies at the same time intervals as those in the RMR trial: engraftment at 12 and 36 months. In that cohort of patients, as in all patients based on 621 biopsies, the CADI score progressed rapidly in the first year (1.4 to 3.3.), and then from 3.3 to 4.3 between 12 and 36 months. The progression over 1236 months was the same as that seen in the SRL-CsA-ST patients with serial biopsies in the RMR study (3.7 to 4.7). The decrease in the CADI score over the same period in the SRL-ST group (3.5 to 3.2) contrasts quite favorably.

Using a design similar to the RMR trial, Stallone and coworkers reported the results of serial engraftment and 12-month protocol-mandated biopsies in 40 patients (20). An imbalance in histologic findings of the baseline biopsies was observed in favor of the SRL-CsA-ST group. This notwithstanding, at 12 months, both the vascular and Banff 97 CAN scores were significantly better in the early CsAwithdrawal group. As could be expected, renal function was also significantly better at 12 months when CsA was withdrawn at month 3 .

Findings from the RMR trial beg the question as to whether the biopsy findings would be even better if a CsA-free regimen had been used from the time of transplantation. Although the CsA exposure in this trial was much lower than that in earlier double-blind trials with sirolimus $(10,21)$, CsA is nephrotoxic even in relatively small doses, and concomitant use of mTOR inhibitors, such as sirolimus, enhances the nephrotoxicity of CsA. A randomized singlecenter study by Flechner and colleagues has shown that adding basiliximab induction to sirolimus-MMF-steroids resulted in significantly better renal function and significantly less interstitial fibrosis at 2 years when compared with a similar CsA-containing regimen (22). Ultimately, a larger multicenter trial will be necessary to confirm whether CNIfree therapy may also be an advantageous context for sirolimus-based therapy.

In summary, the CADI score has proven to be a useful parameter by which to evaluate treatment-related differences in the progression of histologic changes during a large multicenter trial. The serial biopsies obtained in the RMR study were representative of patients completing 3 years of assigned treatment. The results of these biopsies have shown an improvement in renal histology in the early CsA withdrawal group, and therefore, reinforce the advantages of early CsA withdrawal over continuous combined sirolimus-CsA maintenance therapy for the longterm management of renal transplant recipients. Further studies are needed to determine whether the histological advantages noted in the present study are observed when sirolimus is compared directly with CsA without sirolimus, 
and whether the improvements in graft function and histology afforded by CsA elimination can also be extended to patients with higher immunologic risk.

\section{Acknowledgements}

We would like to recognize all of the clinical centers that contributed biopsies. These centers and the investigators are listed in the original publication (12). The authors would like to express their appreciation to Martine GioudPaquet and Eric Nègre of Wyeth Research, Paris, France, for their important contributions to the design and execution the RMR study. This work was sponsored by a grant from Wyeth Research, Collegeville, PA, USA, and Paris, France.

\section{References}

1. Hariharan S, McBride MA, Cherikh WS, Tolleris CB, Bresnahan BA, Johnson CP. Post-transplant renal function in the first year predicts long-term kidney transplant survival. Kidney Int 2002; 62 : 311-318.

2. Paraaskevas S, Kandaswamy R, Humar A et al. Predicting longterm kidney graft survival: can new trials be performed? Transplantation 2003; 75: 1256-1259.

3. Isoniemi H, Taskinen E, Häyry P. Histological chronic allograft damage index accurately predicts chronic renal allograft rejection. Transplantation 1994; 58: 1195-1198.

4. Yilmaz S, Tomlanovich S, Mathew T et al. Protocol core needle biopsy and histologic chronic allograft damage index (CADI) as surrogate end point for long-term graft survival in multicenter studies. J Am Soc Nephrol 2003; 14: 773-779.

5. de Mattos AM, Olyaei AJ, Bennett WM. Nephrotoxicity of immunosuppressive drugs: long-term consequences and challenges for the future. Am J Kid Dis 2000; 35: 333-346.

6. Ojo AO, Held PJ, Port FK et al. Chronic renal failure after transplantation of a nonrenal organ. N Engl J Med 2003; 349: 931940.

7. Rascusen LC, Solez K, Colvin RB et al. The Banff 97 working classification of allograft pathology. Kidney Int 1999; 55: 713-723.

8. DiJoseph JF, Sharma RN, Chang JY. The effect of rapamycin on kidney function in the Sprague-Dawley rat. Transplantation 1992 53: 507-513.

9. Morales JM, Wramner L, Kreis $\mathrm{H}$ et al. Sirolimus does not exhibit nephrotoxicity compared to cyclosporine in renal transplant recipients. Am J Transplant 2002; 2: 436-442.

10. Kahan BD for the Rapamune US Study Group. Efficacy of sirolimus compared with azathioprine for the reduction of acute renal allograft rejection: a randomised multicentre study. Lancet 2000; 356: 194-202

11. Eisen HJ, Tuzcu EM, Dorent $R$ et al. Everolimus for the prevention of allograft rejection and vasculopathy in cardiac-transplant recipients. N Engl J Med 2003; 349: 847-858.

12. Johnson RWG, Kreis H, Oberbauer R, Brattström C, Claesson $\mathrm{K}$, Eris J. Sirolimus allows early cyclosporine withdrawal in renal transplantation resulting in improved renal function and lower blood pressure. Transplantation 2001; 72: 777-786.

13. Oberbauer $R$, Kreis $H$, Johnson RWG et al. Long-term improvement in renal function with sirolimus after early cyclosporine withdrawal in renal transplant recipients: 2-year results of the Rapamune Maintenance Regimen Study. Transplantation 2003; 76 : 364-370.

14. Kreis H, Oberbauer R, Campistol JM et al. Long-term benefits with sirolimus-based therapy after early cyclosporine withdrawal. J Am Soc Nephrol 2004; 15: 809-817.

15. Yilmaz S, Nutley $M$, Taskinen $E$ et al. Chronic allograft damage index as a surrogate marker for chronic allograft rejection. In: Trull A, Price C, Demers LM (eds): Biomarkers of Disease. Cambridge, UK: Cambridge University Press, 2002: 433-441.

16. Nankivell BJ, Gruenewald SM, Allen RD, Chapman JR. Predicting glomerular filtration rate after kidney transplantation. Transplantation 1995; 59: 1683-1689.

17. Solez K, Axelsen RA, Benediktsson $\mathrm{H}$ et al. International standardization of criteria for the histologic diagnosis of renal allograft rejection: the Banff working classification of kidney transplant pathology. Kidney Int 1993; 44: 411-422.

18. Legendre C, Thervet E, Skhiri $\mathrm{H}$ et al. Histologic features of chronic allograft nephropathy revealed by protocol biopsies in kidney transplant recipients. Transplantation 1998; 65: 1506-1509.

19. Solez K, Vincenti F, Filo RS, for The FK506 Kidney Transplant Study Group. Histopathologic findings from 2-year protocol biopsies from a U.S. multicenter kidney transplant trial comparing tacrolimus versus cyclosporine. Transplantation 1998; 66: 17361740 .

20. Stallone G, Di Paolo S, Schena A et al. Early withdrawal of cyclosporine A improves 1-year kidney graft structure and function in sirolimus-treated patients. Transplantation 2003; 75: 998-1003.

21. MacDonald AS for the Rapamune Global Study Group. A worldwide, phase III, randomized, controlled, safety and efficacy study of a sirolimus/cyclosporine regimen for prevention of acute rejection in recipients of primary mismatched renal allografts. Transplantation 2000; 71: 271-280.

22. Flechner SM, Burke JT, Cook DJ et al. A randomized prospective trial of sirolimus vs cyclosporine in renal transplantation: renal function and histology at two years. Am J Transplant 2003; 3 : 450. 
Copyright of American Journal of Transplantation is the property of Blackwell Publishing Limited and its content may not be copied or emailed to multiple sites or posted to a listserv without the copyright holder's express written permission. However, users may print, download, or email articles for individual use. 